Recepción: 15/01/2019

Aceptación: 19/03/2019

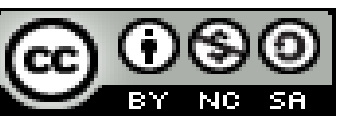

Ciencias económicas y empresariales

Publicación: 05/05/2019

Artículo de investigación

\title{
La influencia del Merchandising en el punto de venta: Caso práctico Supermercados "Mi Caserita"
}

\section{The influence of Merchandising at the point of sale: Case study Supermarkets "Mi Caserita"}

\section{A influência do merchandising no ponto de venda: estudo de caso Supermercados "Mi Caserita"}

\author{
Jorge Francisco Abril-Flores ${ }^{\mathrm{I}}$ \\ jf.abril@uta.edu.ec \\ Julio César Zurita-Altamirano ${ }^{\mathrm{II}}$ \\ jzurita@pucesa.edu.ec \\ Juan Enrique Ramos-Guevara ${ }^{\text {III }}$ \\ je.ramos@uta.edu.ec \\ Martha Liliana Albán-Bautista IV \\ martha.alban@utc.edu.ec
}

Correspondencia:jf.abril@uta.edu.ec

\footnotetext{
${ }^{\text {I } M a g i ́ s t e r ~ e n ~ D o c e n c i a ~ y ~ C u r r i ́ c u l o ~ p a r a ~ l a ~ E d u c a c i o ́ n ~ S u p e r i o r, ~ M a g i ́ s t e r ~ e n ~ A d m i n i s t r a c i o ́ n ~ d e ~ E m p r e s a s ~ M e n c i o ́ n ~}$ Planeación, Doctor en Contabilidad y Auditoría, Licenciado en Contabilidad y Auditoría Contador Público, Docente Universidad Técnica de Ambato, Ambato, Ecuador.

II Magíster en Administración de Empresas Mención Planeación, Ingeniero Comercial con Mención en Marketing, Docente Pontificia Universidad Católica del Ecuador Sede Ambato, Ambato, Ecuador.

III Magíster en Gerencia Financiera Empresarial, Magíster en Seguridad e Higiene Industrial y Ambiental, Ingeniero en Alimentos, Docente Universidad Técnica de Ambato, Ambato. Ecuador.

IV Magíster en Ciencias de la Educación Mención en Gestión Educativa y Desarrollo Social, Ingeniera en Ciencias Administrativas Especialización Contabilidad y Auditoría CPA, Licenciada en Ciencias de la Educación Especialidad Contabilidad y Computación, Docente Universidad Técnica de Cotopaxi, Cotopaxi. Ecuador.
} 


\section{Resumen}

La investigación se enfoca en determinar el impacto del merchandising en el punto de venta y su influencia en la toma de decisiones. El estudio se realizó a los clientes del supermercado Mi Caserita ubicado en Huachi Chico Ambato. Los resultados obtenidos son el punto de partida para evaluar y mejorar el merchandising en el punto de venta. Esta investigación se les aplicó a 383 clientes, una encuesta para valorar diferentes aspectos de marketing y funcionamiento general. El nivel de investigación se orienta a dos tipologías de alcances. Por un lado, está la investigación descriptiva, porque se analizaron las variables sobre las realidades en el punto de venta, y se realizó una interpretación de forma imparcial sobre las observaciones ejecutadas. También es de tipo cuantitativa ya que se utilizaron instrumentos de medición, y estadísticos para la tabulación de los resultados. De acuerdo a los datos obtenidos el merchandising sí influye en los consumidores en la toma de decisiones. Los involucrados manifiestan que factores como la luz, el aroma, la distribución de los productos en las góndolas, la variedad de productos y la realización de promociones motivan a las compras no programadas, lo que va de la mano al realizar el supermercado una buena gestión del punto de venta y posicionamiento de los artículos dentro del establecimiento comercial. Otras de las conclusiones es que la atención es reconocida como débil, debiendo mejorarla para obtener mejores resultados. De manera consecutiva se sugiere el uso de uniforme para dar una mejor imagen de la empresa.

Palabras clave: Merchandising; supermercado; punto de venta; decisiones; clientes.

\section{Abstract}

The research focuses on determining the impact of merchandising at the point of sale and its influence on decision making. The study was conducted to the clients of the My Caserita supermarket located in Huachi Chico Ambato. The results obtained are the starting point to evaluate and improve the merchandising at the point of sale. This investigation took place in the supermarket "My Caserita" for which 383 customers were applied to a survey to assess different aspects of marketing and general operation. The level of research is oriented to two types of scopes. On the one hand, there is descriptive research, because the variables on the realities at the point of sale were analyzed, and an unbiased interpretation was made of the observations made. It is also quantitative since measurement instruments were used, and statistics for the tabulation of 
the results. According to the data obtained, merchandising does influence consumers in decision making. Those involved say that factors such as light, aroma, the distribution of products in the gondolas, the variety of products and the realization of promotions motivate unscheduled purchases, which goes hand in hand when making the supermarket a good management of the point of sale and positioning of the articles within the commercial establishment. Another conclusion is that attention is recognized as weak, and should be improved to obtain better results. Consecutively, the use of uniform is suggested to give a better image of the company.

Keywords: Merchandising; supermarket; point of sale; decisions; customers.

\section{Resumo}

A pesquisa se concentra em determinar o impacto do merchandising no ponto de venda e sua influência na tomada de decisão. O estudo foi realizado para clientes do supermercado $\mathrm{Mi}$ Caserita localizado em Huachi Chico Ambato. Os resultados obtidos são o ponto de partida para avaliar e melhorar o merchandising no ponto de venda. Esta investigação ocorreu no supermercado "Mi Caserita", para o qual 383 clientes foram aplicados a uma pesquisa para avaliar diferentes aspectos de marketing e operação geral. O nível de pesquisa é orientado para dois tipos de escopos. Por um lado, há pesquisa descritiva, porque as variáveis sobre as realidades no ponto de venda foram analisadas, e uma interpretação imparcial foi feita das observações feitas. Também é quantitativo, pois foram utilizados instrumentos de medição e estatísticas para a tabulação dos resultados. De acordo com os dados obtidos, o merchandising influencia os consumidores na tomada de decisão. Os envolvidos dizem que fatores como a luz, o aroma, a distribuição de produtos nas gôndolas, a variedade de produtos e a realização de promoções motivam compras não programadas, que andam de mãos dadas ao fazer do supermercado uma boa gestão. Do ponto de venda e posicionamento dos artigos dentro do estabelecimento comercial. Outra conclusão é que a atenção é reconhecida como fraca e deve ser melhorada para obter melhores resultados. Consecutivamente, o uso de uniformes é sugerido para dar uma melhor imagem da empresa.

Palavras-chave: Merchandising; supermercado ponto de venda; decisões; clientes. 


\section{Introducción}

El comercio minorista a través de cadenas locales ha incrementado su presencia con productos de consumo masivo. Su lucha constante por mantener una posición significativa ante las grandes cadenas de comercio. Se trata del reto constante del incremento de ventas, diseñando estrategias que hagan atractivo el punto de venta por medio del merchandising. En base a esta herramienta el cliente se informa, comunica y persuade a los clientes.

Es probable que el merchandising surgió en el comienzo de las actividades de comercialización. Desde el comienzo de las actividades del trueque, el ser humano ha expuesto sus productos en ciertos mostradores rústicos. En esta época, la exhibición de los productos se realizaba en materiales rústicos como telas, costales, entre otras cosas (Jiménez Marín, 2018). En la evolución del merchandising, intervinieron varios aspectos propios de aquella época. Los mercaderes evidenciaron la necesidad de exhibir sus productos de una manera adecuada, de modo que los clientes pueden acceder con facilidad y apreciar antes de realizar una compra (Burbano \& Morales, 2010).

El estudio del merchandising como disciplina, da inicio cuando surge grandes almacenes y supermercados (Ramirez \& Alférez, 2014). La noción básica de los almacenes es facilitar y dinamizar la compra. La dinámica del negocio en autoservicios consistía en que el cliente pueda escoger sus productos directamente y no esperar de algún servidor del almacén para escoger entre los distintos productos. El primer almacén surgió en Francia en 1878 (Palomares Borja, 2013). Después aparecieron más almacenes populares en EEUU en 1978.

El vocablo merchandising proviene del término anglosajón, conformada de dos términos: merchandising significa mercadería y el sufijo significa acción. Son actividades que estimulan la decisión de compra en el punto de venta (Ramírez \& Alferez, 2014).

Se refiere a administrar el área física para obtener el máximo rendimiento posible evitando la errónea ubicación de las marcas en el establecimiento (Castillo, 2004). Es el conjunto de métodos y técnicas para ubicar productos a la vista del cliente, incitarlo a la compra aun cuando no se encuentre un vendedor (Gómez \& Rubio, 2006). 
Es poner a la vista los productos a los clientes de manera que se puedan agrupar con un diseño de superficie de ventas llamativo (Parra, Lheirmie, \& José, 2008). Se debe tomar a consideración la ubicación de los productos y la forma adecuada de presentarlos al público. (Ramirez \& Alférez, 2014).

Compuesto por cuatro ejes importantes, estudio de mercado encargado de segmentar clientes y realizar análisis de la competencia, gestión lineal optimizar el espacio, gestión del surtido que colabora con la selección, análisis y determinación del surtido (Rodriguéz, 2017). La animación del punto de venta a través de promociones o publicidad en el lugar de venta (Castillo, 2004).

Para Ramírez \& Alferez (2014) la parte visual busca la comercialización del producto. Resalta las características y atributos para hacerlo más atractivo en el punto de venta (Pinzón, 2015). La parte de gestión sirve de justificativo para tomar decisiones estratégicas (Rodriguéz, 2017). Para (Burbano \& Morales, 2010) trabaja en áreas operativas para gestionar la demanda y oferta de productos.

\section{EI Merchandising de Gestión abarca 4 pilares:}

Para recopilar información, se debe desarrollar un estudio de forma constante en relación al comportamiento de los consumidores y retener o modificar la oferta en función de las peticiones de los clientes (Burbano \& Morales, 2010). Es vital para la toma de decisiones puesto que estudia la oferta y demanda, canales de distribución, el precio y la logística de distribución (Ramirez \& Alférez, 2014).

La gestión del espacio coloca los productos de forma adecuada para provocar la venta alcanzando mayor rentabilidad (Pinzón, 2015). La distribución del espacio físico debe estar dado en secciones, la distancia entre góndolas, y otros estímulos que realcen el lugar (Ramirez \& Alférez, 2014).

La gestión del surtido selecciona los productos más adecuado para el público objetivo, y determinar la amplitud, profundidad y coherencia de cada producto (Bobes \& Valdéz, 2014). Para Pinzón (2015) tiene como fin proporcionar a los clientes variedad de productos entre marca, precio y calidad. El surtido de los productos en las góndolas es esta dado por cada supermercado

\section{6}

Pol. Con. (Edición núm. 33) Vol. 4, No 5, mayo 2019, pp. 323-337, ISSN: 2550 - 682X 
(Ramírez \& Alferez, Modelo conceptual para determinar el impacto del Merchandasing visual en la toma de decisiones de decisiones, 2014).

La comunicación usa todo tipo de promociones o información en el punto de venta Gómez y Rubio (2006). El correcto uso de la publicidad en el lugar de venta contribuye a fortalecer la imagen corporativa, e influyen en el crecimiento de las ventas de los productos que comercializa (Ramírez \& Alferez, Modelo conceptual para determinar el impacto del Merchandasing visual en la toma de decisiones de decisiones, 2014).

En el desarrollo de las actividades de un merchandising efectivo están involucradas varias partes: fabricantes, distribuidores, comerciantes, promotores, entre otros (Ardila, 2016). De las cuales destacan los fabricantes y distribuidores, los cuales, si bien no tienen aspectos coincidentes, si son actores fundamentales (Palacios, 2008).

El merchandising del fabricante es el conjunto de técnicas comerciales elaboradas por los productores para hacer de sus artículos más atractivos hacia sus clientes (Bastos, 2006). Rodríguez \& Dopico (1998) acotan que los fabricantes necesitan utilizar canales de distribucion indirectos para hacer llegar sus productos al cliente final, es así que cuando este artículo entra en la cadena de distribución se pierde el manejo sobre los mismos, por lo cual su principal meta es obtener una presencia adecuada en el punto de comercialización.

Del mismo modo, Mora (2010) afirma que la efectividad del merchandising para el fabricante se da cuando se conoce directamente el punto de venta, como se ve la categoría de productos, los colores y formas que destacan y el contexto que está alrededor del productos.

Se debe delimitar un contraste entre el merchandising que realiza el fabricante y el distribuidor, ya que mientras el primero prioriza defender su marca e incrementar su posicionamiento, el segundo se enfoca en consolidar su clientela e incrementar las ventas (Fernández, 2005).

Los distribuidores realizar un conjunto de prácticas que se aplican sobre los productos y establecimientos, enfocadas a incrementar la rentabilidad de los puntos de venta activos (Bastos, 2006). Sus funciones se reparten en la organización del establecimiento, organización de las secciones, elección del surtido, como se colocan los productos dentro del establecimiento, entre otros (Tellado, 2009). De igual manera Fajado (2011) aporta que el distribuidor debe conocer las 
gamas de producto y los elementos que el fabricante le proporciona (carteles, regalos, góndolas, etc) y usarlos de manera adecuada.

Por otro lado, los distribuidores son los encargados de atraer a los potenciales clientes que accedan al local comercial a través de los componentes de la infraestructura del local: fachada, mobiliarios, corredores, zonas calientes y frias, vallas, rótulos, etc (Ramirez \& Alférez, 2014). Esto ya que los distribuidores tiene poca o nula influencia en cuanto a la calidad del producto, el empaque, los costos, publicidad o dostribucion de los articulos que comercualiza en sus locales (Gómez \& Rubio, 2006) y deben buscar formas de hacerle atractivo a un producto y asi incentivar a su compra.

Atención, Interés, Deseo y Acción, este modelo propuesto por Elmo Lewis en 1986 hace referencia a los efectos en el momento de la venta (Ryte, 2017). La Atención busca en el consumidor entretenimiento y despertar curiosidad; el Interés en lo curioso y mediante la comunicación poder persuadirlo; el Deseo de obtener un producto y la Acción es la adquisición del producto o servicio (Ramirez \& Alférez, 2014).

El modelo AIDA en el marketing persigue que sus elementos se desarrollen de tal manera que la decisión de compra sea inmediata, complaciendo al consumidor y más que todo generar réditos para la empresa (Espinoza, 2017). Este modelo tiene años de aplicación, pero depende del vendedor y más que todo del punto de venta para lograr su objetivo (Ramírez C. , 2013).

\section{Modelo Nicosia}

Es uno de los primeros modelos del comportamiento del consumidor en el proceso de decisión de compra. Sus elementos son la predisposición, las actitudes y las motivaciones. La predisposición del individuo en el momento de la compra; las actitudes son impulsos débiles de las personas y las motivaciones son fuertes impulsos que incitan a la acción de compra (Ramírez \& Alferez, 2014).

Francisco Nicosia presenta su modelo a través de un diagrama de flujo dónde interactúan las variables de forma consecutiva (Manzuoli, 2007). La comunicación y los anuncios buscan respuesta en los consumidores e influir en sus mentes. De este modo la comunicación atrae la

\section{8}

Pol. Con. (Edición núm. 33) Vol. 4, No 5, mayo 2019, pp. 323-337, ISSN: 2550 - 682X 
atención del consumidor, el mismo que después la evalúa y dar lugar a alternativas, luego se motiva por la compra y cierra el negocio.

\section{Modelo Howard Sheth}

El modelo Howard-Sheth es el proceso que pretende exponer la conducta y el comportamiento del comprador o consumidor al escoger la marca de un determinado bien o servicio (Ramírez \& Alferez, 2014). En la toma de decisión con respecto a un producto interviene tres elementos. Este modelo, es un marco de referencia de análisis para una teoría más amplia del comportamiento del consumidor (Jiménez Marín, 2018).

Este modelo propone tres pasos antes de la decisión de adquirir un producto: (1) Solución profunda de problemas, el comprador o interesado tiene poca información sobre las marcas de los productos y todavía no puede desarrollar juicios bien definidos y estructurados. (2) Solución limitada de problemas, los razonamientos de selección están bien determinados, pero el comprador no tiene muy claro sobre cuál grupo de marcas será el más apto. (3) Comportamiento de respuesta habitual, los compradores tienen criterios de selección bien definidos y también inclinaciones firmes por una marca (Farley \& Ring, 2011).

La investigación se enfoca en determinar el impacto del merchandising en el punto de venta y su influencia en la toma de decisiones. Determinar cómo estos factores ayudan en la toma de decisiones, fundamentar teóricamente y la obtención de resultados. El estudio se realizada a los clientes de Mi Caserita ubicación en Huachi Chico. Estos resultados son el punto de partida para evaluar y mejorar el merchandising en el punto de venta.

\section{Metodología}

El nivel de investigación se orienta a dos tipologías de alcances. Por un lado está la investigación descriptiva, porque se analizaron las variables sobre las realidades en el punto de venta, y se realizó una interpretación de forma imparcial sobre las observaciones ejecutadas. El instrumento empleado para la encuesta consto con un total de 7 preguntas y fue utilizado el método de la escala de Likert para la formulación de las preguntas. También es de tipo cuantitativa ya que se utilizaron instrumentos de medición, y estadísticos para la tabulación de los resultados. La población total del estudio la conforma la población económicamente activa de Ambato, que 
según el Censo de Estadística y población 2010 está conformado por 161240 personas. Aplicando las formulas necesarias la muestra para este estudio fue de 383 personas.

\section{Resultados}

A continuación, se presentan los resultados obtenidos de la aplicación del instrumento a las 383 personas, sobre la influencia del Merchandising en el punto de venta. Las preguntas fueron formuladas a través de la escala de Likert, que es un instrumento ampliamente utilizado, ya que trata de medir la intensidad con que los encuestados se sientan identificados con respecto a la pregunta planteada.

1. ¿Está usted de acuerdo en que la publicidad pueda tener influencia en el punto de venta?

Grafico 1. Influencia de la publicidad en el punto de venta

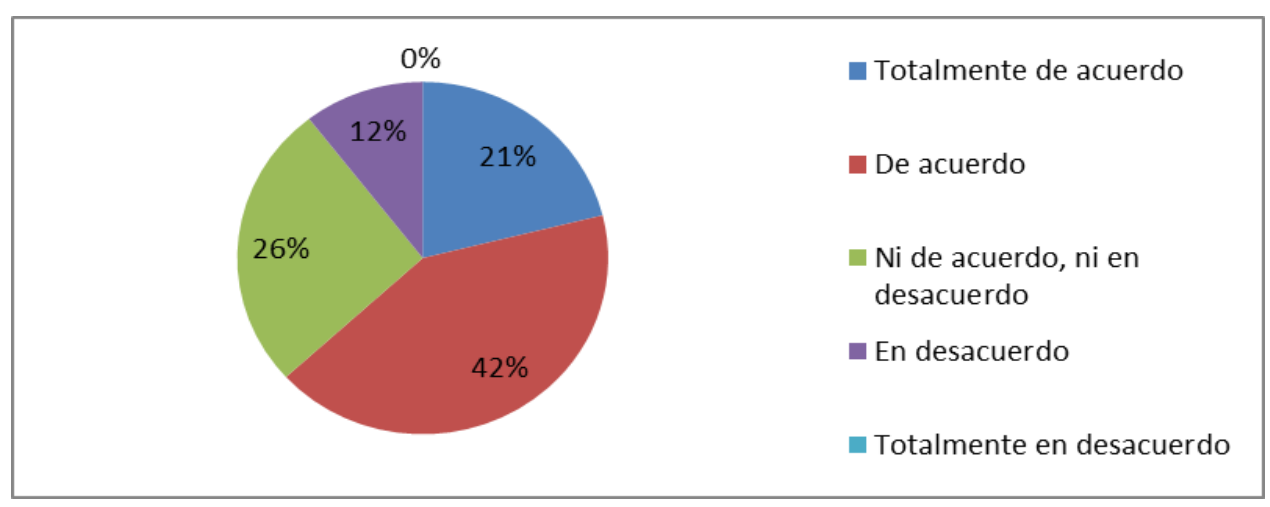

Fuente: Elaboración Propia

El 63\% de los encuestados está de acuerdo en que la publicidad influye en el punto de venta, el $26 \%$ no parece prestarle mucha atención al tema de la publicidad y el $12 \%$ no está de acuerdo en que la publicidad influye en el punto de venta. Esto quiere decir que supermercados Mi Caserita debe invertir en publicidad para promocionarse como supermercado y los productos y servicios que ofrece. 
2. ¿Está usted de acuerdo en que la imagen visual (iluminación y decoración) de supermercados Mi Caserita es importante?

Grafico 2. Importancia de la imagen visual de supermercados Mi Caserita

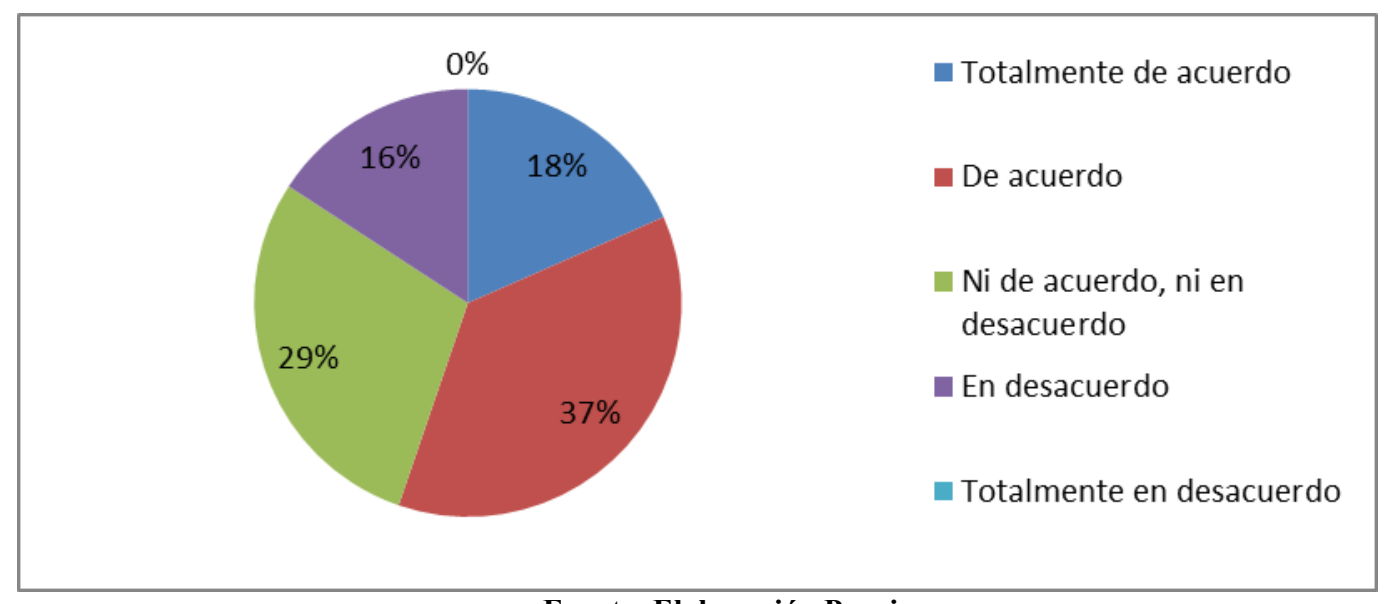

Fuente: Elaboración Propia

El 55\% de los encuestados considera que la imagen visual de un local o almacén de venta de productos es muy importante a la hora de la captación de posibles clientes por medio de la utilización y puesta en práctica del merchandising. El 29\% considera que no es importante y el $16 \%$ está en desacuerdo que la imagen visual de supermercados Mi Caserita, sea importante. Esto quiere decir que supermercados Mi Caserita debe invertir en la infraestructura de local para hacerla más amigable a los clientes, en este sentido, la opinión de la clientela es muy importante, es por ello que supermercados Mi caserita por medio de buzones de sugerencia puede emplear mecanismos de comunicación con la clientela para determinar que mejoras o cambios debe hacer en su infraestructura. 
3. En general, ¿qué tan satisfecho o insatisfecho te sientes con respecto a la atención brindada por los trabajadores de Supermercados Mi Caserita?

Grafico 3. Grado de satisfacción con la atención brindada por el personal de supermercados Mi Caserita

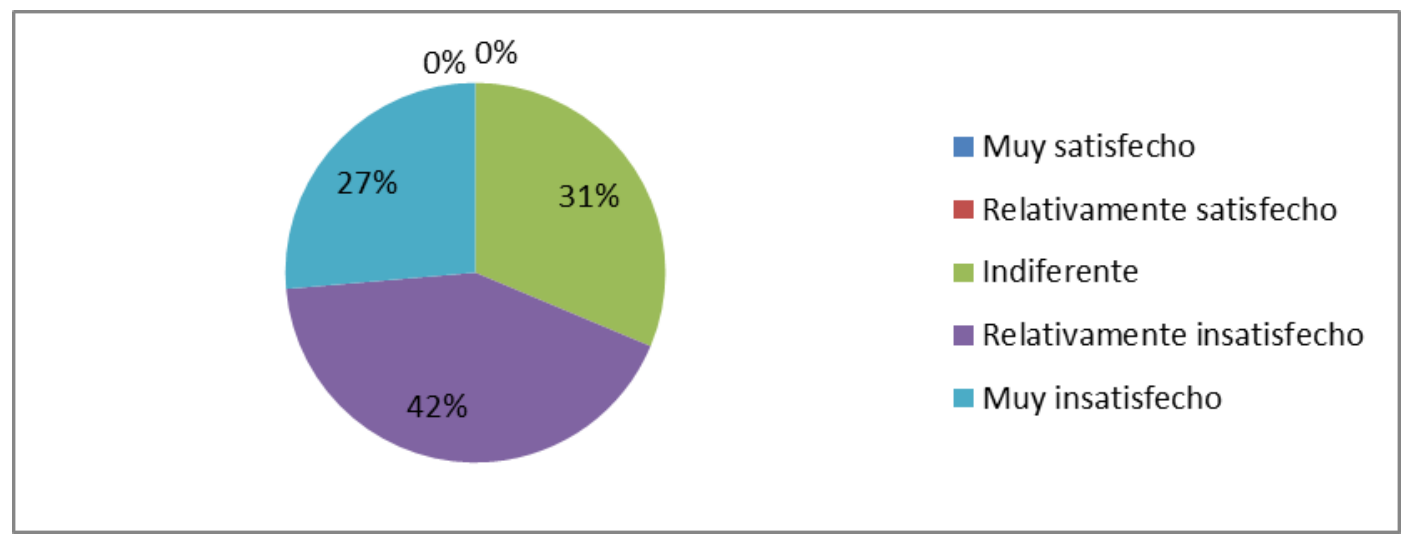

Fuente: Elaboración Propia

El 71\% de los encuestados considera que el servicio al cliente que brinda Supermercados Mi Caserita debe mejorar, ya que tienen una percepción muy negativa al respecto. El $31 \%$ considera que el servicio al cliente le es indiferente. Esto pone en evidencia los esfuerzos que debe emprender el supermercado para elevar al máximo nivel de satisfacción el servicio al cliente que brindan, para ello es importante capacitar al personal sobre el trato que se le debe dar a los clientes y buscar en su defecto trabajadores que ya tengan experiencia en atención al cliente.

4. ¿Cree usted que es muy útil una adecuada ubicación de los productos dentro de supermercados Mi Caserita?

\section{Grafico 4. Utilidad de una adecuada ubicación de los productos}

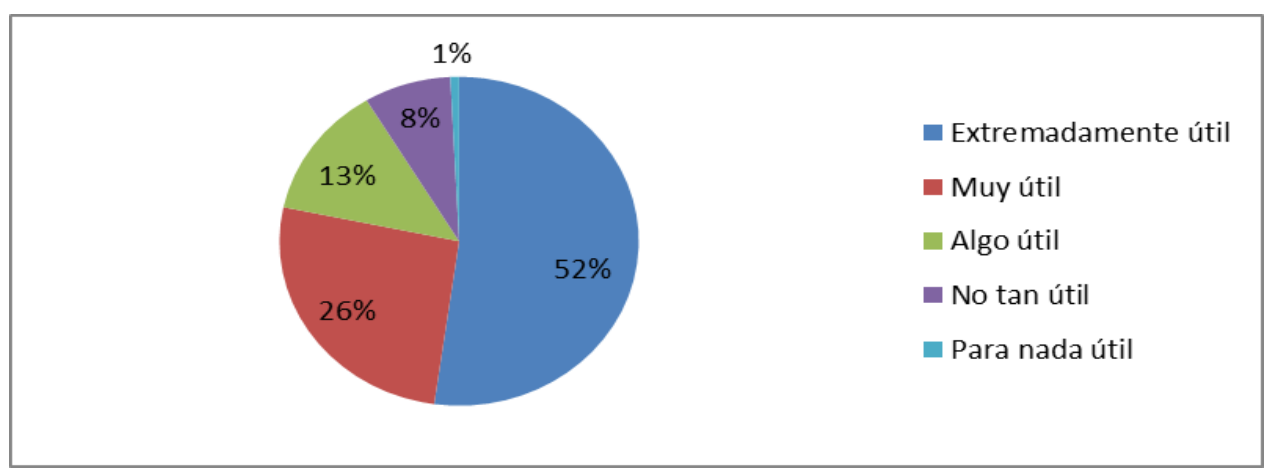

Fuente: Elaboración Propia

Pol. Con. (Edición núm. 33) Vol. 4, No 5, mayo 2019, pp. 323-337, ISSN: 2550 - 682X 
Con los resultados obtenidos, el 78\% de los clientes involucrados, afirman que es muy útil el uso de la logística o ubicación de los productos que ofrece Supermercados Mi Caserita, mientras que el $13 \%$ no ve la viabilidad de la colocación de los productos y el $9 \%$ no le parece útil colocar estratégicamente los productos. Se concluye que la ubicación de los productos, es un punto clave percibido por los clientes que frecuentan el Supermercado Mi Caserita, hay muchos estudios que se han enfocado en determinar qué es lo primero que hace un cliente al entrar a un supermercado, y la organización de los productos a exhibir depende de ello.

\section{En general, ¿qué tan de acuerdo o desacuerdo se siente usted con la colocación de los productos en góndolas en los Supermercados Mi Caserita?}

\section{Grafico 5. Presentación de productos en estanterías}

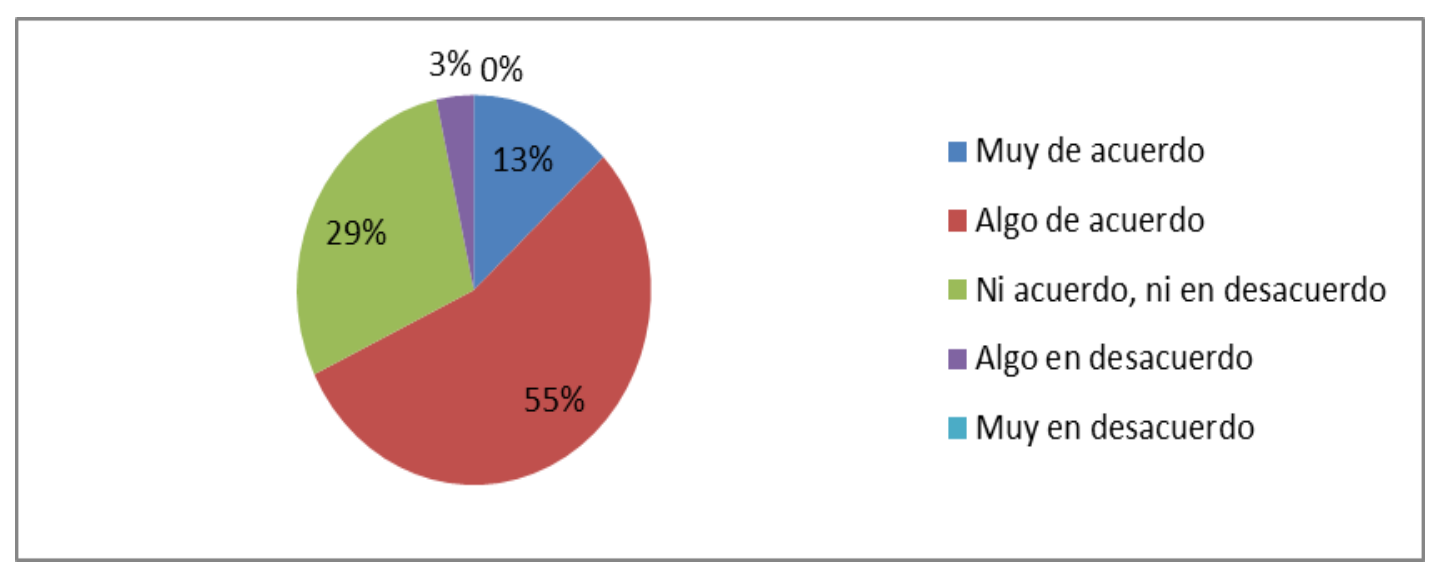

Fuente: Elaboración Propia

Con los resultados obtenidos, los involucrados mencionan que la presentación de los productos en góndolas es buena con el 68\%, seguida del 29\% que mantiene una posición neutral y un 3\% que no está de acuerdo. Supermercados Mi Caserita debe enfocarse en exhibir sus productos en góndolas, ya que las mismas, por estrategias de mercado, generalmente están al principio de las filas de las estanterías de los supermercados. 
6. ¿Está usted de acuerdo en que supermercados Mi Caserita debe tener otros métodos de exhibición de productos?

Grafico 6. Importancia de tener otros métodos de exhibición de productos dentro del supermercado

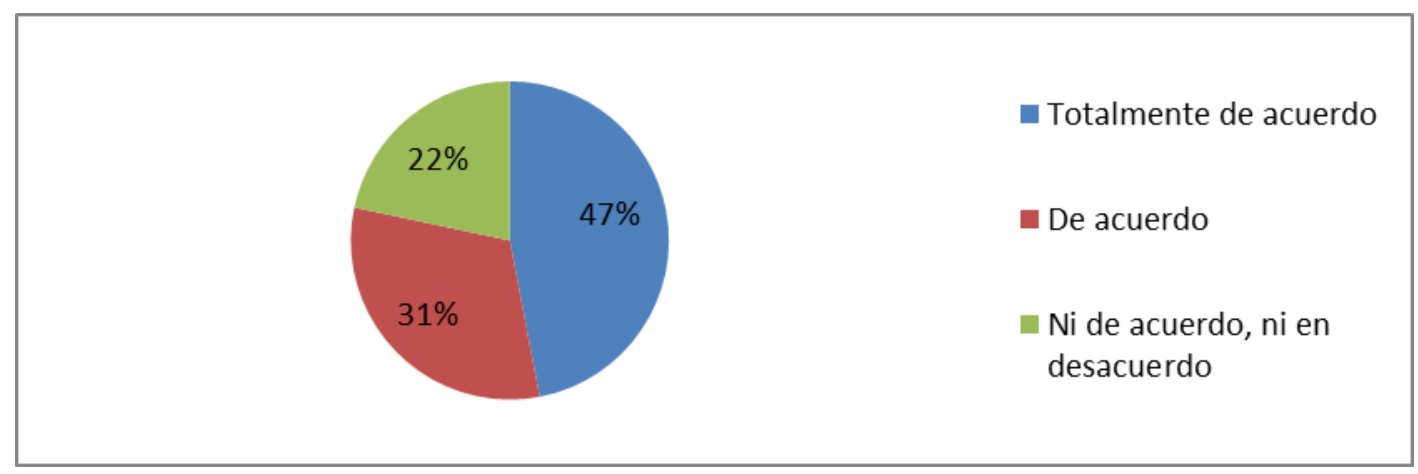

Fuente: Elaboración Propia

En lo que corresponde a la variedad para exhibir los productos, el $78 \%$ está de acuerdo en que supermercados Mi Caserita debe emplear otros métodos de exhibición de sus productos, el 22\% no le da mucha importancia.

7. ¿Cree usted que es muy útil la promoción en eventos de productos de supermercados Mi Caserita?

Grafico 7. Grado de utilidad de promoción de productos de supermercados Mi Caserita

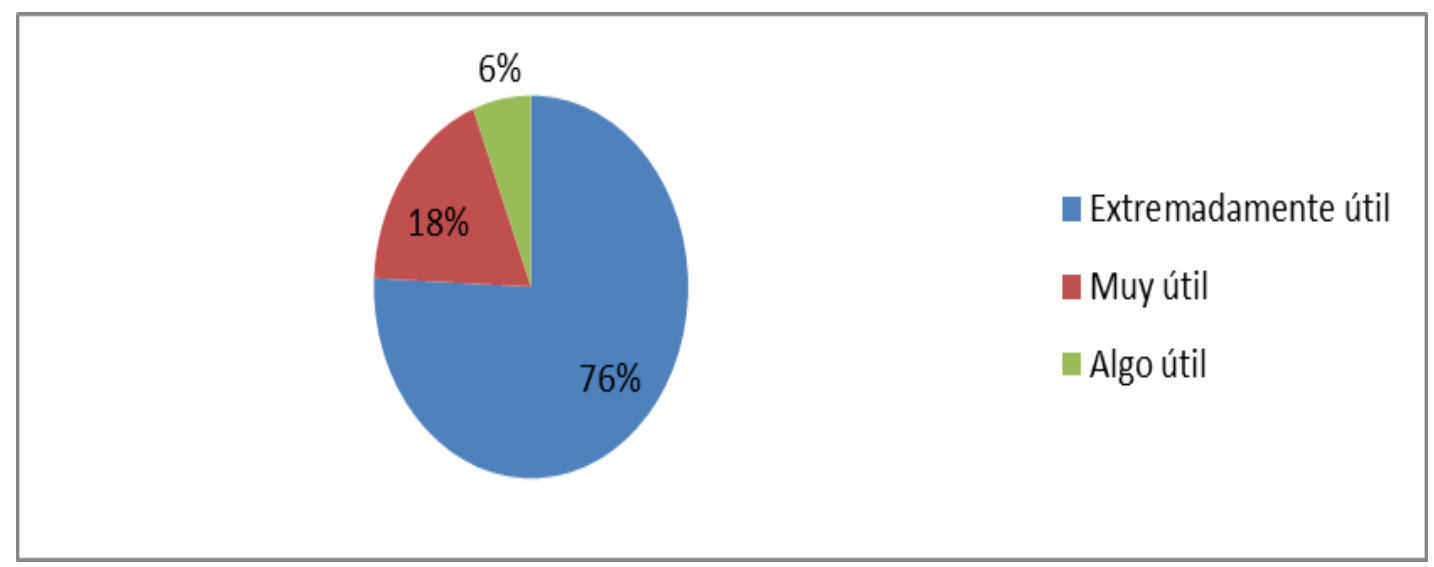

Fuente: Elaboración Propia 
El 94\% de los clientes encuestados afirma estar de acuerdo con las promociones para incitar la compra de productos dentro de supermercados Mi Caserita, mientras que el 6\% menciona que no existe ningún cambio en su decisión de compra.

\section{Conclusiones}

De acuerdo a los datos obtenidos el merchandising sí influye en los consumidores en la toma de decisiones. Los involucrados manifiestan que factores como la luz, el aroma, la distribución de los productos en las góndolas, la variedad de productos y la realización de promociones motivan a las compras no programadas.

Más allá del espacio físico y estímulos en el punto de venta, se debe tener claro que la generación de experiencias en los supermercados crea sentimientos memorables y agradables, como consecuencia se obtendrá mayor impacto en las decisiones de compra.

Cabe recalcar que el servicio al cliente es un factor importante que acompaña al merchandising ya que influye en la toma de decisiones de los consumidores, además de marcar presencia en sus mentes, en los resultados obtenidos la atención es reconocida como débil, debiendo mejorarla para obtener mejores resultados. De manera consecutiva se sugiere el uso de uniforme para dar una mejor imagen de la empresa.

\section{Referencias Bibliográficas}

Ardila, W. (2016). Las prácticas de merchandising en las tiendas de barrio. Bogotá D.C: Ediciones Universidad Central.

Bastos, A. (2006). Merchandising y animación del punto de venta: Manual básico de merchandising. Madrid: Ideaspropias.

Bobes, A., \& Valdéz, P. (2014). Servicio logístico al cliente en empresas de servicios: procedimiento para su diseño. Economía y Desarrollo, 184-192.

Burbano, E., \& Morales, R. (2010). Incidencia en la logística del marketing. Guillermo de Ockhan, 37-54. 
Castillo, A. (2004). Gestión por categorias, una integración entre fabricnates y distribuidores. España: Netbiblo.

Espinoza, R. (11 de Abril de 2017). ¿Qué es el modelo AIDA en el marketing. Madrid.

Farley, J., \& Ring, W. (2011). Models of Buyer Behavior, Chapter 8: Deriving an Empirically Testable Version of the Howard-Sheth Model of Buyer Behavior. Estados Unidos : Marketing Classics Press.

Fernández, S. (2005). Técnicas de negociación. Habilidades para negociar con éxito. Vigo: Ideaspropias Editorial.

Gómez, M., \& Rubio, N. (2006). Gestión minoristas de marcas de distribuidor productos y merchandising. ICE, 157-174.

Gómez, M., \& Rubio, N. (2008). Gestión de minotistas de las marcas de distribuidor: Producto y Merchandising. TENDENCIAS DE LA DISTRIBUCIÓN COMERCIAL EN EL ÁMBITO INTERNACIONAL, 157-174.

Jiménez Marín, G. (2018). La gestión profesional del merchandising. Barcelona : Editorial UOC.

Lafuente, C., \& Marín, A. (2017). Metodologías de la investigación en las ciencias sociales. Revista Escuela de Administración de negocios: Fases, fuentes y selección de técnicas, 518.

Manzuoli, J. (2007). Una visión renovadora sobre el proceso de decisión de compra. Revista electrónico FCE.

Palacios, J. (2008). Técnicas avanzadas de negociación. La Coruña: Netbiblio S. L.

Palomares Borja, R. (2013). Merchandising. Teoría, práctica y estrategia. Madrid : ESIC Editorial.

Parra, F., Lheirmie, C., \& José, R. (2008). Distribución comericial. Madrid: ESIC. 
Pinzón, S. (2015). La logística y la gestión de la cadena de suministro como estrategias de marketing. Investigación y Ciencia, 80-81.

Ramírez, C. (2013). Modelo conceptual para determinar el impacto del merchandising visual en la toma de decisiones de compra en el punto de venta. Red de Revistas Científicas de América Latina y el Caribe, España y Portugal , 1-27.

Ramirez, C., \& Alférez, L. (2014). El Merchandising en el establecimiento virtual, una aproximación al diseño y usabilidad. Pensamiento y gestión, 1-27.

Ramírez, C., \& Alferez, L. (2014). Modelo conceptual para determinar el impacto del Merchandasing visual en la toma de decisiones de decisiones. Pensamiento y Gestión, 127.

Ramírez, F., \& Zwerg, A. (2012). Metodología de la investigación:más que una receta . ADminister, 91-111.

Rodriguéz, C. (2017). Una nueva definición de la logística interna y forma de evaluar la misma. Revista chilena de ingenieria, 264-276.

Ryte. (2017). Modleo AIDA. Obtenido de Ryte Wiki- La enciclopedia del Marketing Digital: https://es.ryte.com/wiki/Modelo_AIDA

Tellado, F. (17 de Febrero de 2009). Lugar de la negociación. Obtenido de Técnicas de Negociación: https://tellado.es/descargas/negociacion/tecnicas-negociacion.pdf 